\title{
Pseudo 1-homogeneous distance-regular graphs
}

\author{
Aleksandar Jurišić • Paul Terwilliger
}

Received: 18 November 2005 / Accepted: 14 December 2007 / Published online: 4 January 2008

(C) Springer Science+Business Media, LLC 2008

\begin{abstract}
Let $\Gamma$ be a distance-regular graph of diameter $d \geq 2$ and $a_{1} \neq 0$. Let $\theta$ be a real number. A pseudo cosine sequence for $\theta$ is a sequence of real numbers $\sigma_{0}, \ldots, \sigma_{d}$ such that $\sigma_{0}=1$ and $c_{i} \sigma_{i-1}+a_{i} \sigma_{i}+b_{i} \sigma_{i+1}=\theta \sigma_{i}$ for all $i \in\{0, \ldots$, $d-1\}$. Furthermore, a pseudo primitive idempotent for $\theta$ is $E_{\theta}=s \sum_{i=0}^{d} \sigma_{i} A_{i}$, where $s$ is any nonzero scalar. Let $\hat{v}$ be the characteristic vector of a vertex $v \in V \Gamma$. For an edge $x y$ of $\Gamma$ and the characteristic vector $w$ of the set of common neighbours of $x$ and $y$, we say that the edge $x y$ is tight with respect to $\theta$ whenever $\theta \neq k$ and a nontrivial linear combination of vectors $E \hat{x}, E \hat{y}$ and $E w$ is contained in $\operatorname{Span}\{\hat{z} \mid$ $z \in V \Gamma, \partial(z, x)=d=\partial(z, y)\}$. When an edge of $\Gamma$ is tight with respect to two distinct real numbers, a parameterization with $d+1$ parameters of the members of the intersection array of $\Gamma$ is given (using the pseudo cosines $\sigma_{1}, \ldots, \sigma_{d}$, and an auxiliary parameter $\varepsilon$ ).

Let $S$ be the set of all the vertices of $\Gamma$ that are not at distance $d$ from both vertices $x$ and $y$ that are adjacent. The graph $\Gamma$ is pseudo 1-homogeneous with respect to $x y$ whenever the distance partition of $S$ corresponding to the distances from $x$ and $y$ is equitable in the subgraph induced on $S$. We show $\Gamma$ is pseudo 1-homogeneous with respect to the edge $x y$ if and only if the edge $x y$ is tight with respect to two distinct real numbers. Finally, let us fix a vertex $x$ of $\Gamma$. Then the graph $\Gamma$ is pseudo 1-homogeneous with respect to any edge $x y$, and the local graph of $x$ is connected if and only if there is the above parameterization with $d+1$ parameters $\sigma_{1}, \ldots, \sigma_{d}, \varepsilon$ and the local graph of $x$ is strongly regular with nontrivial eigenvalues $a_{1} \sigma /(1+\sigma)$ and $\left(\sigma_{2}-1\right) /\left(\sigma-\sigma_{2}\right)$.
\end{abstract}

\footnotetext{
A. Jurišić $(\bowtie)$

Faculty of Computer and Informatic Sciences, University of Ljubljana, Ljubljana, Slovenia e-mail: ajurisic@valjhun.fmf.uni-lj.si

P. Terwilliger

Department of Mathematics, University of Wisconsin-Madison, Madison, WI 53706-1388, USA
} 
Keywords Distance-regular graphs · Primitive idempotents · Cosine sequence · Locally strongly regular $\cdot 1$-homogeneous property · Tight distance-regular graph · Pseudo primitive idempotent · Tight edges · Pseudo 1-homogeneous

\section{Introduction}

Let $\Gamma=(X, R)$ be a distance-regular graph with vertex set $X$, edge set $R$ and diameter $d$. For $u \in X$ and $i \in\{0, \ldots, d\}$ we denote by $\Gamma_{i}(u)$ the set of vertices at distance $i$ from $u$. Let $x, y \in X$ be two adjacent vertices and let us consider the distance partition of the vertex set $X$ corresponding to $x$ and $y$. Denoting the intersection $\Gamma_{i}(x) \cap \Gamma_{j}(y)$ by $D_{i}^{j}(x, y)$ or just by $D_{i}^{j}$, we can describe this partition in the following way

$$
\pi=\left\{D_{i}^{j} \mid i, j=0, \ldots, d\right\} .
$$

Note that $D_{i}^{j}=\emptyset$ when $|i-j|>1$ by the triangle inequality and $D_{i}^{i+1} \neq \emptyset \neq D_{i+1}^{i}$ for $i=1, \ldots, d-1$. Let us assume $a_{1} \neq 0$. Then we have also $D_{i}^{i} \neq \emptyset$ for $1 \leq i<d$ (see Figure 4.1 and Section 5). Finally, $D_{d}^{d}=\emptyset$ if and only if $a_{d}=0$. Next we study this partition from an algebraic point of view.

Let $\operatorname{Mat}_{X}(\mathbb{R})$ denote the $\mathbb{R}$-algebra consisting of all matrices with entries in $\mathbb{R}$, whose rows and columns are indexed by $X$ and let $A \in$ Mat $_{X}(\mathbb{R})$ be the adjacency matrix of $\Gamma$. Let $V=\mathbb{R}^{X}$ be the vector space consisting of all column vectors with entries in $\mathbb{R}$, whose coordinates are indexed by $X$. Observe that $\operatorname{Mat}_{X}(\mathbb{R})$ acts on $V$ by left multiplication. For a subset $S \subseteq X$ let its characteristic vector be an element of $V$, whose coordinates equal 1 if they correspond to the elements of $S$ and 0 otherwise. Let $w_{i j}$ be the characteristic vector of $D_{i}^{j}$ and $W=W(x, y)=\operatorname{Span}\left\{w_{i j} \mid i, j=\right.$ $0, \ldots, d\}$. Hence

$$
\operatorname{dim} W= \begin{cases}3 d & \text { if } a_{d} \neq 0, \\ 3 d-1 & \text { if } a_{d}=0 .\end{cases}
$$

One way to define a vertex partition of $\Gamma$ to be equitable is to require that the span of characteristic vectors of all the parts of $\pi$ be $A$-invariant, cf. [5, Lem. 5.2.1]. We say that the graph $\Gamma$ is $\mathbf{1 - h o m o g e n e o u s ~ w i t h ~ r e s p e c t ~ t o ~} x y$ whenever the partition $\pi$ defined in (1) is equitable. Let us assume this is the case, i.e., $A W \subseteq W$. Since the Bose-Mesner algebra of $\Gamma$, denoted by $\mathcal{M}$, is the subalgebra of $\operatorname{Mat}_{X}(\mathbb{R})$ generated by $A$, we also have $\mathcal{M} W=W$.

Let $\hat{v}$ denote the characteristic vector of $\{v\}$, where $v$ is a vertex of $\Gamma$. Set $w=w_{11}$ and $H=H(x, y)=\operatorname{Span}\{\hat{x}, \hat{y}, w\}$. We study the action of $\mathcal{M}$ on $H$. Since $\Gamma$ is 1 homogeneous, an easy induction argument implies that the action of $\mathcal{M}$ on $H$ generates the whole $W$, i.e., $\mathcal{M} H=W$, where $\mathcal{M} H$ means $\operatorname{Span}\{m h \mid m \in \mathcal{M}, h \in H\}$. Since the primitive idempotents $E_{0}, E_{1}, \ldots, E_{d}$ form a basis for the Bose-Mesner algebra $\mathcal{M}$, we have in view of $E_{i} E_{j}=\delta_{i j} E_{i}$ for all $i, j \in\{0, \ldots, d\}$,

$$
\mathcal{M} H=\sum_{i=0}^{d} E_{i} H \quad \text { (direct sum). }
$$


Note that $\operatorname{dim}\left(E_{0} H\right)=1$. Furthermore, for all $i \in\{1, \ldots, d\}$ we have $3 \geq \operatorname{dim}\left(E_{i} H\right)$ and, since the vectors $E_{i} \hat{x}$ and $E_{i} \hat{y}$ are linearly independent, cf. [7, Lem. 2.7], we also have $\operatorname{dim}\left(E_{i} H\right) \geq 2$. We say the edge $x y$ is tight with respect to $E_{i}$ (or to the corresponding eigenvalue $\theta$ ) whenever the vectors $E_{i} \hat{x}, E_{i} \hat{y}, E_{i} w$ are linearly dependent. So the edge $x y$ is tight with respect to $E_{i}$ if and only if $\operatorname{dim}\left(E_{i} H\right)=2$. By [7, Thm.5.2], $\operatorname{dim}\left(E_{i} H\right)=2$ implies $i \in\{1, d\}$, so

$$
\operatorname{dim}(\mathcal{M} H)=3 d+1-t,
$$

where $t$ denotes the number of nontrivial eigenvalues of $\Gamma$ with respect to which $\operatorname{dim}\left(E_{i} H\right)=2$, and $t \in\{0,1,2\}$. However, the graph $\Gamma$ is 1 -homogeneous with respect to $x y$, so we have $t \neq 0$ by (2) and $t=2$ if and only if $a_{d}=0$.

Let $\Gamma$ be a distance-regular graph of diameter $d \geq 3$, and eigenvalues $\theta_{0}>\theta_{1}>$ $\cdots>\theta_{d}$. It was shown in Jurišić, Koolen and Terwilliger [7] that the intersection numbers $a_{1}, b_{1}$ satisfy the following inequality

$$
\left(\theta_{1}+\frac{k}{a_{1}+1}\right)\left(\theta_{d}+\frac{k}{a_{1}+1}\right) \geq-\frac{k a_{1} b_{1}}{\left(a_{1}+1\right)^{2}},
$$

called the Fundamental Bound, and $\Gamma$ was defined to be tight whenever it is not bipartite, and equality holds in (4). Tight graphs have been characterized in a number of interesting ways. See Jurišić, Koolen and Terwilliger [7], Pascasio [8] and Go \& Terwilliger [4]. We collect some of their results in the following theorem.

Theorem 1.1 Let $\Gamma$ be a distance-regular graph with diameter $d \geq 3$, eigenvalues $\theta_{0}>\cdots>\theta_{d}$ and $\theta, \theta^{\prime}$ a pair of distinct nontrivial eigenvalues. Then $\Gamma$ is tight if and only if any of the following conditions is true.

(i) $a_{1} \neq 0$ and there exists an edge of $\Gamma$ which is tight with respect to both $\theta_{1}, \theta_{d}$.

(ii) There exists $x \in X$ such that the local graph of $x$ is connected, strongly-regular with eigenvalues $a_{1},-1-b_{1} /\left(1+\theta_{d}\right)$ and $-1-b_{1} /\left(1+\theta_{1}\right)$.

(iii) $a_{1} \neq 0, a_{d}=0$, and $\Gamma$ is 1-homogeneous with respect to at least one edge.

(iv) There exist real scalars $\xi_{0}, \ldots, \xi_{d}, \varepsilon, h$ such that $\xi_{0}=1, \xi_{d-1}=\xi_{1} \xi_{d}, \varepsilon \neq-1$, $k=c_{d}=h\left(\xi_{1}-\varepsilon\right) /\left(\xi_{1}-1\right)$, and for all $i \in\{1, \ldots, d-1\}$ we have

$$
b_{i}=h \frac{\left(\xi_{i-1}-\xi_{1} \xi_{i}\right)\left(\xi_{i+1}-\varepsilon \xi_{i}\right)}{\left(\xi_{i-1}-\xi_{i+1}\right)\left(\xi_{i+1}-\xi_{i}\right)}, \quad c_{i}=h \frac{\left(\xi_{i+1}-\xi_{1} \xi_{i}\right)\left(\xi_{i-1}-\varepsilon \xi_{i}\right)}{\left(\xi_{i+1}-\xi_{i-1}\right)\left(\xi_{i-1}-\xi_{i}\right)} .
$$

Moreover, if $\Gamma$ is tight, then the above conditions are satisfied for all edges and vertices of $\Gamma,\left\{\theta, \theta^{\prime}\right\}=\left\{\theta_{1}, \theta_{d}\right\}$, the sequence $\xi_{0}, \ldots, \xi_{d}$ is a cosine sequence corresponding to $\theta_{1}$ or $\theta_{d}$ and $|\varepsilon|=\left(k^{2}-\theta_{1} \theta_{d}\right) /\left(k\left(\theta_{1}-\theta_{d}\right)\right)$.

Other characterizations include the existence of tight cosine sequences, a property of primitive idempotents and the existence of short irreducible $T(x)$-modules with endpoint 1 for a vertex $x$. By extending this theory to so-called pseudo 1-homogeneous graphs, we are extending the approach that was developed in Jurišić, Koolen and Terwilliger [7]. Our theory is satisfied by examples that are not necessarily tight, for example the halved cubes of odd diameter and the complement of the Higman-Sims graph. 
This paper is organized as follows. After preliminaries about cosine sequences in Section 2, we introduce in Section 3 pseudo cosine sequences as a sequence of real numbers that satisfy all but the last recurrence relation of the cosine sequences. Then we introduce pseudo primitive idempotents that correspond to pseudo cosine sequences and were first defined in Terwilliger and Weng [11]. In Section 4 we define what it means for an edge to be tight with respect to a real number distinct from the valency of the graph, establish some basic properties and, in Section 5, we study some combinatorial consequences of this property.

Let $\Gamma$ be a distance-regular graph with diameter $d \geq 2$, that is not triangle-free. In Section 6 we introduce an auxiliary parameter $\varepsilon$ and in Section 7 we study the case when an edge of $\Gamma$ is tight with respect to two distinct real numbers, in which case we give a parameterization with $d+1$ parameters of the members of intersection array of $\Gamma$ (namely with the pseudo cosines $\sigma_{1}, \ldots, \sigma_{d}$ and $\varepsilon$ ).

Let $S$ be the set of vertices of $\Gamma$ that are not at distance $d$ from both vertices $x$ and $y$. The graph $\Gamma$ is pseudo 1-homogeneous with respect to $\boldsymbol{x} \boldsymbol{y}$ whenever the distance partition of $S$ corresponding to the distances from $x$ and $y$ is equitable in the subgraph induced on $S$. Finally, we give two characterizations of the pseudo 1homogeneous property with respect to an edge in Section 9. First, we show that the graph $\Gamma$ is pseudo 1-homogeneous with respect to the edge $x y$ if and only if the edge $x y$ is tight with respect to two distinct real numbers. For the second, we first define the graph $\Gamma$ to be pseudo 1-homogeneous with respect to a vertex $\boldsymbol{x}$ whenever it is pseudo 1-homogeneous with respect to all edges incident with $x$. Curtin and Nomura [3] have characterized graphs with this property in terms of their subconstituent algebra. We offer another characterization: the distance-regular graph $\Gamma$ is pseudo 1homogeneous with respect to a vertex $x$ such that the local graph of $x$ is connected if and only if $\Gamma$ allows the above parameterization with $d+1$ parameters $\sigma_{1}, \ldots, \sigma_{d}, \varepsilon$ and the local graph of $x$ is strongly regular with a nontrivial eigenvalues $a_{1} \sigma /(1+\sigma)$ and $\left(\sigma_{2}-1\right) /\left(\sigma-\sigma_{2}\right)$. We conclude the paper with an open problem.

\section{Preliminaries}

In this section, we review some definitions and basic concepts that have not been covered in the introduction. See the books of Bannai and Ito [1], Godsil [5], or Brouwer, Cohen and Neumaier [2] for more background information.

Let $\Gamma=(X, R)$ be a graph. Let $\Omega$ denote a subset of $X$. By the subgraph of $\Gamma$ induced on $\Omega$, we mean the graph with vertex set $\Omega$, and edge set consisting of those edges of $\Gamma$ that have both ends in $\Omega$. The subgraph induced on $\Gamma(u)=\Gamma_{1}(u)$ is called the local graph of $u$ and will be denoted by $\Delta(u)$. We set $k_{i}=\left|\Gamma_{i}(u)\right|$, and for $v \in \Gamma_{i}(u)$ also $c_{i}=\left|\Gamma_{i-1}(u) \cap \Gamma(v)\right|, a_{i}=\left|\Gamma_{i}(u) \cap \Gamma(v)\right|, b_{i}=\left|\Gamma_{i+1}(u) \cap \Gamma(v)\right|$ (note that the last three sets partition the local graph of $v$ ). Assume from now on $\Gamma$ is distance-regular. Then $a_{i}, b_{i}, c_{i}$ and $k_{i}$ are independent of choice of $u$ and $v \in \Gamma_{i}(u)$. Furthermore, for each $i, j, h \in\{0, \ldots, d\}$ there exists a constant $p_{i j}^{h}$ such that $p_{i j}^{h}=$ $\left|\Gamma_{i}(x) \cap \Gamma_{j}(y)\right|$ for any two vertices $x$ and $y$ at distance $h$. Observe $\Gamma$ is regular with valency $k=b_{0}=c_{i}+a_{i}+b_{i}$ and the subgraph induced on $\Gamma_{i}(u)$ is regular with valency $a_{i}$ and $k_{i}$ vertices. 
For each integer $i, 0 \leq i \leq d$, let $A_{i}$ be the $i$ th distance matrix in $\operatorname{Mat}_{X}(\mathbb{R})$, defined with $\left(A_{i}\right)_{x y}=1$, if $\partial(x, y)=i$ and 0 otherwise. Then $A=A_{1}$. Observe $A_{0}=I$, $A_{0}+\cdots+A_{d}=J$, where $J$ is the all 1's matrix, $A_{i}^{t}=A_{i}$ for all $i \in\{0, \ldots, d\}$, where $t$ denotes transposition, and for all $i, j \in\{0, \ldots, d\}$ we have $A_{i} A_{j}=\sum_{h=0}^{d} p_{i j}^{h} A_{h}$. In particular, by the triangle inequality, we have $A A_{j}=c_{j+1} A_{j+1}+a_{j} A_{j}+b_{j-1} A_{j-1}$ for all $j \in\{0, \ldots, d\}$, where $A_{-1}=A_{d+1}=0$ and $b_{-1}, c_{d+1}$ are unspecified. Using the above properties one can readily show that matrices $A_{0}, \ldots, A_{d}$ form a basis for the Bose-Mesner algebra $\mathcal{M}$. For $i \in\{0, \ldots, d\}$ let $f_{i} \in \mathbb{R}[\lambda]$ denote the unique polynomial with degree $i$ such that $f_{i}(A)=A_{i}$. Then $f_{0}=1$ and $\lambda f_{i}=$ $c_{i+1} f_{i+1}+a_{i} f_{i}+b_{i-1} f_{i-1}$ for all $i \in\{0, \ldots, d-1\}$, with $f_{-1}=0$. For $0 \leq i \leq d$ we define $p_{i}=f_{0}+\cdots+f_{i}$.

By Bannai and Ito [1, p.59, p.64], the algebra $\mathcal{M}$ has a second basis $E_{0}, \ldots, E_{d}$ such that $E_{0}=|X|^{-1} J, E_{0}+\ldots+E_{d}=I, E_{i}^{t}=E_{i}$ for all $i \in\{0, \ldots, d\}$, and $E_{i} E_{j}=\delta_{i j} E_{i}$ for all $i, j \in\{0, \ldots, d\}$. The matrices $E_{0}, \ldots, E_{d}$ are known as the primitive idempotents of $\Gamma$. We refer to $E_{0}$ as the trivial idempotent. Let $\theta_{0}, \ldots, \theta_{d}$ be the real numbers satisfying $A=\sum_{i=0}^{d} \theta_{i} E_{i}$. Observe $A E_{i}=E_{i} A=\theta_{i} E_{i}$ for all $i \in\{0, \ldots, d\}$, and that $\theta_{0}, \ldots, \theta_{d}$ are distinct as $A$ generates $\mathcal{M}$. It follows from the above properties that $\theta_{0}=k$, and it is known that $-k \leq \theta_{i}<k$ for all $i \in\{1, \ldots, d\}$, see for example Bannai and Ito [1, p. 197]. We refer to $\theta_{i}$ as the eigenvalue of $\Gamma$ associated with $E_{i}$, and call $\theta_{0}$ the trivial eigenvalue. For each integer $i, 0 \leq i \leq d$, let $m_{i}$ denote the rank of $E_{i}$. We refer to $m_{i}$ as the multiplicity of $E_{i}$ (or $\theta_{i}$ ) and observe $m_{0}=1$.

We now recall the cosines. Let $\theta$ be an eigenvalue of $\Gamma$, and $E$ the associated primitive idempotent. Let $\sigma_{0}, \ldots, \sigma_{d}$ be the real numbers satisfying

$$
E=\frac{m}{|X|} \sum_{i=0}^{d} \sigma_{i} A_{i},
$$

where $m$ is the multiplicity of $\theta$. Taking the trace in (5), we find $\sigma_{0}=1$. We often abbreviate $\sigma=\sigma_{1}$. We refer to $\sigma_{i}$ as the $i$ th cosine of $\Gamma$ with respect to $\theta$ (or $E$ ), and call $\sigma_{0}, \ldots, \sigma_{d}$ the cosine sequence of $\Gamma$ associated with $\theta$ (or $E$ ). The cosines can be interpreted as follows. We endow $V=\mathbb{R}^{X}$ with the Euclidean inner product satisfying $\langle u, v\rangle=u^{t} v$ for $u, v \in V$, and note that $\{\hat{x} \mid x \in X\}$ is an orthonormal basis for $\mathbb{R}^{X}$. The following is an easy application of properties of primitive idempotents that we mentioned above. See [7, Lemma 2.1].

Lemma 2.1 Let $\Gamma=(X, R)$ be a distance-regular graph with diameter $d$. Let $E$ be a primitive idempotent and $\sigma_{0}, \ldots, \sigma_{d}$ its cosine sequence. Then for all integers $i \in\{0, \ldots, d\}$, and for all vertices $x$ and $y$ at distance $i$, the following holds.

(i) $\langle E \hat{x}, \hat{y}\rangle=\langle\hat{x}, E \hat{y}\rangle=\langle E \hat{x}, E \hat{y}\rangle=m \sigma_{i}|X|^{-1}$, where $m$ denotes the multiplicity of $E$.

(ii) The cosine of the angle between the vectors $E \hat{x}$ and $E \hat{y}$ equals $\sigma_{i}$.

Let $\Gamma$ be a distance-regular graph with diameter $d$ and let $\theta, \sigma_{0}, \ldots, \sigma_{d}$ be any complex numbers. Then, by Brouwer, Cohen and Neumaier [2, Sect. 4.1.B], $\theta$ is an 
eigenvalue of $\Gamma$ and $\sigma_{0}, \ldots, \sigma_{d}$ is the associated cosine sequence if and only if $\sigma_{0}=1$, and we have for all $i \in\{0, \ldots, d\}$ the following recursion

$$
c_{i} \sigma_{i-1}+a_{i} \sigma_{i}+b_{i} \sigma_{i+1}=\theta \sigma_{i},
$$

where $\sigma_{-1}$ and $\sigma_{d+1}$ are indeterminates.

\section{Pseudo primitive idempotents}

Let $\Gamma$ be a distance-regular graph with diameter $d$. Suppose $\theta$ is a real number (not necessarily an eigenvalue of $\Gamma$ ) and that the scalars $\sigma_{0}, \ldots, \sigma_{d}$ are defined by $\sigma_{0}=1$ and the above recursion (6) for all $i \in\{0, \ldots, d-1\}$, or equivalently by $\sigma_{0}=1$, $k \sigma=\theta$, and

$$
c_{i}\left(\sigma_{i-1}-\sigma_{i}\right)-b_{i}\left(\sigma_{i}-\sigma_{i+1}\right)=k(\sigma-1) \sigma_{i} \text { for all } i \in\{1, \ldots, d-1\} .
$$

Then we call $\sigma_{0}, \ldots, \sigma_{d}$ the pseudo cosine sequence for $\theta$. In particular, we have $\sigma_{1}=\theta / k$ and $\sigma_{2}=\left(\theta^{2}-a_{1} \theta-k\right) /\left(k b_{1}\right)$ when $d \geq 2$. If $\theta \neq k$, then we say that the pseudo cosine sequence for $\theta$ is nontrivial. It is straightforward to verify that $f_{i}(\theta)=\sigma_{i} k_{i}$ for all $i \in\{0, \ldots, d\}$. Therefore, for all $i \in\{0, \ldots, d-1\}$ we have, by the recursion $p_{i+1}=f_{i+1}+p_{i}$ and (7), also

$$
p_{i}(\theta)=k_{i} b_{i} \frac{\sigma_{i}-\sigma_{i+1}}{k-\theta} .
$$

This can be used in the following result, which describes three instances, where the pseudo cosine sequence is especially well behaved.

Lemma 3.1 Let $\Gamma$ be a distance-regular graph with diameter $d \geq 2$, and eigenvalues $\theta_{0}>\cdots>\theta_{d}$. Let $\theta$ be a real number and assume $k \neq \theta \geq \theta_{1}$ or $\theta \leq \theta_{d}$. Let $\sigma_{0}, \ldots, \sigma_{d}$ be a pseudo cosine sequence for $\theta$.

(i) If $\theta>k$ then $\sigma_{0}<\cdots<\sigma_{d}$.

(ii) If $k>\theta \geq \theta_{1}$ then $\sigma_{0}>\cdots>\sigma_{d}$.

(iii) If $\theta \leq \theta_{d}$ then $(-1)^{i} \sigma_{i}>0$ for all $i \in\{0, \ldots, d\}$.

Proof Let $i(0 \leq i \leq d-1)$ be an integer. By Terwilliger [9, Lem. 4.5], we get for $\theta \geq$ $\theta_{1}$ that $p_{i}(\theta)>0$. Now the statements (i) and (ii) follow immediately by (8). Finally, the statement (iii) follows directly from [2, Prop. 4.1.1(ii)] and [2, Prop. 4.1.1(i)] that holds for any real number (and not just an eigenvalue).

Let $\theta$ be a real number. By a pseudo primitive idempotent for $\theta$ we mean

$$
E_{\theta}=s \sum_{i=0}^{d} \sigma_{i} A_{i},
$$

where $s$ is any nonzero scalar and $\sigma_{0}, \ldots, \sigma_{d}$ is the pseudo cosine sequence for $\theta$ defined above. While $E_{\theta}^{2}$ is in general not necessarily equal to $E_{\theta}$, we can still derive at least one property of primitive idempotents mentioned in the above Lemma 2.1. 
Lemma 3.2 Let $\Gamma$ be a distance-regular graph with diameter $d$. Let $\sigma_{0}, \ldots, \sigma_{d}$ be a pseudo cosine sequence, let s be a nonzero scalar and $E$ the corresponding pseudo primitive idempotent. Then for all integers $i \in\{0, \ldots, d\}$, and for all vertices $x$ and $y$ at distance $i$

$$
\langle E \hat{x}, \hat{y}\rangle=\langle\hat{x}, E \hat{y}\rangle=s \sigma_{i}
$$

Proof By the definition of a pseudo primitive idempotent, see (9), we have

$$
\langle\hat{x}, E \hat{y}\rangle=s \sum_{h=0}^{d} \sigma_{h}\left\langle\hat{x}, A_{h} \hat{y}\right\rangle=s \sigma_{i} .
$$

Similarly we obtain also $\langle E \hat{x}, \hat{y}\rangle=s \sigma_{i}$.

The following result provides an alternative view of pseudo primitive idempotents.

Lemma 3.3 Let $\Gamma$ be a distance-regular graph with diameter $d$. Let $\theta$ be a real number. Let $E$ be an element of the Bose-Mesner algebra. Then $E$ is a pseudo primitive idempotent for $\theta$ if and only if $E \neq 0$ and

$$
(A-\theta I) E \in \operatorname{Span}\left(A_{d}\right) .
$$

Proof Suppose $E \neq 0$ and that (10) holds. Set $E=\sum_{i=0}^{d} \sigma_{i} A_{i}$ for some real numbers $\sigma_{i}$. Then there exists $\gamma \in \mathbb{R}$, such that

$\gamma A_{d}=(A-\theta I) E=(A-\theta I) \sum_{j=0}^{d} \sigma_{j} A_{j}=\sum_{j=0}^{d} A_{j}\left(c_{j} \sigma_{j-1}+a_{j} \sigma_{j}+b_{j} \sigma_{j+1}-\theta \sigma_{j}\right)$.

Hence $c_{j} \sigma_{j-1}+a_{j} \sigma_{j}+b_{j} \sigma_{j+1}=\theta \sigma_{j}$ for all $j \in\{0,1, \ldots, d-1\}$. The number $\sigma_{0}$ is nonzero, as otherwise $\sigma_{i}=0$ for all $i \in\{1, \ldots, d\}$ and thus also $E=0$. Therefore, if we divide all the members of the sequence $\sigma_{0}, \ldots, \sigma_{d}$ by $\sigma_{0}$, we obtain a pseudo cosine sequence for $\theta$. Thus $E$ is a pseudo primitive idempotent. The converse is straightforward.

\section{Tight edges}

We generalize the definition of an edge being tight with respect to an eigenvalue of a graph to the definition of an edge being tight with respect to any real number.

Let $\Gamma$ be a distance-regular graph with diameter $d \geq 2, a_{1} \neq 0$. Let us define the scalar $f=f(x, y)$ to be the average valency of the complement of the subgraph induced on $D_{1}^{1}=D_{1}^{1}(x, y)$. Then the subgraph induced on $D_{1}^{1}$ has $a_{1}\left(a_{1}-1-f\right) / 2$ edges. Moreover, there are $a_{1} f$ edges connecting vertices in $D_{1}^{1}$ with vertices in $D_{1}^{2}$, and $a_{1}\left(b_{1}-f\right)$ edges connecting vertices in $D_{1}^{1}$ with vertices in $D_{2}^{2}$. Therefore, $0 \leq f \leq \min \left\{a_{1}-1, b_{1}\right\}$. Moreover, $f=0$ if and only if the set $D_{1}^{1}$ induces a clique, 

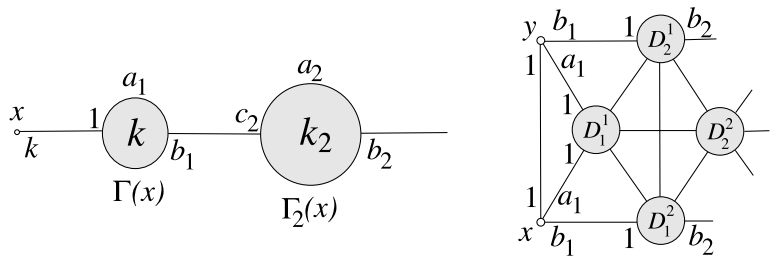

Fig. 1 Local distance partitions of the graph $\Gamma$, the left corresponding to a vertex $x$ and the right corresponding to an edge $x y$. In the left figure we put in the circles the number of their vertices, while in the right one we put in the corresponding names of the sets. The number beside edges connecting two cells indicate how many neighbours a vertex from the closer cell has in the other cell. The number beside a cell is the valency of the graph induced by the vertices of the cell

$f=a_{1}-1$ if and only if the graph induced on $D_{1}^{1}$ has no edges, and $f=b_{1}$ if and only if the graph induced on $D_{1}^{2}(x, y)$ has no edges.

Let $\Gamma$ be a distance-regular graph with diameter $d \geq 2$ and $a_{1} \neq 0$. Let $\theta$ be a nontrivial eigenvalue of $\Gamma$ and $E$ the corresponding primitive idempotent. Then the determinant of the matrix of inner products for vectors $E \hat{x}, E \hat{y}$ and $E w$ is nonnegative, and this inequality translates to the following bound on $f$ :

$$
(k+\theta)(1+\theta) f \leq b_{1}\left(k+\theta\left(a_{1}+1\right)\right),
$$

see Jurišić et al. [7, Lemma 3.3]. Equality is attained in (11) if and only if the vectors $E \hat{x}, E \hat{y}$ and $E w$ are linearly dependent. Therefore, the edge $x y$ is tight with respect to an eigenvalue $\theta$ whenever equality holds in (11). We will generalize the property of an edge being tight with respect to an eigenvalue. To do this we need to introduce one more matrix. For $i, j \in\{0, \ldots, d\}$ let $E_{i j}^{*}$ be a diagonal matrix in $\operatorname{Mat}_{X}(\mathbb{R})$ with

$$
\left(E_{i j}^{*}\right)_{z z}= \begin{cases}1, & \text { if } z \in D_{i}^{j}(x, y) \\ 0, & \text { otherwise. }\end{cases}
$$

Then $E_{i j}^{*} V=\operatorname{Span}\left\{\hat{z} \mid z \in D_{i}^{j}(x, y)\right\}$.

Definition 4.1 Let $\Gamma$ be a distance-regular graph with diameter $d \geq 2$ and $a_{1} \neq 0$. Let $\theta$ be a real number, $E$ the corresponding pseudo primitive idempotent and let $x y$ be an edge of $\Gamma$. We say the edge $x y$ is tight with respect to $\theta$ whenever

(i) $\theta \neq k$, and

(ii) a nontrivial linear combination of vectors $E \hat{x}, E \hat{y}$ and $E w$ is contained in $E_{d d}^{*} V$.

Let $\theta$ be a nontrivial eigenvalue and $x y$ an edge of $\Gamma$. Then the edge $x y$ is tight with respect to $\theta$ in the original sense if and only if the edge $x y$ is tight with respect to $\theta$ by Definition 4.1. Since the "only if" part is obvious, let us show the "if" part. Let $E$ be the primitive idempotent corresponding to $\theta$ and suppose the condition (ii) holds. Let $\Delta$ denote a nontrivial linear combination of vectors $\hat{x}, \hat{y}$ and $w$. Then the assumptions $\langle E \Delta, \hat{x}\rangle=0,\langle E \Delta, \hat{y}\rangle=0$ and $\langle E \Delta, \hat{z}\rangle=0$ for each $z \in D_{1}^{1}(x, y)$ imply $\langle E \Delta, \Delta\rangle=0$. Since $E^{2}=E$ we have $\langle E \Delta, E \Delta\rangle=0$ and finally $E \Delta=0$.

We now obtain a result that is related to [7, Cor. 3.4(iii)]. 
Lemma 4.2 Let $\Gamma$ be a distance-regular graph with diameter $d \geq 2$ and $a_{1} \neq 0$. Let $\theta$ be a real number and $E$ the corresponding pseudo primitive idempotent. Suppose an edge $x y$ is tight with respect to $\theta$. Then $\theta \neq-k$. Let $\sigma$ be the corresponding first cosine and observe $\sigma \neq-1$. Then

$$
E w-\frac{a_{1} \sigma}{1+\sigma}(E \hat{x}+E \hat{y}) \in E_{d d}^{*} V .
$$

Proof By assumption, there exists real scalars $\alpha, \beta, \gamma$, not all zero, such that

$$
\alpha E \hat{x}+\beta E \hat{y}+\gamma E w \in E_{d d}^{*} V
$$

Since $a_{1} \neq 0$ there exists $u \in D_{1}^{1}$. Let $\sigma_{0}, \ldots, \sigma_{d}$ be the pseudo cosine sequence for $\theta$. Evaluating the $x, y$ and $u$ coordinates in the above line, by Lemma 3.2 and $d \geq 2$, we obtain the following system of linear equations

$$
\begin{aligned}
& 0=\alpha+\beta \sigma+\gamma \sigma a_{1}, \\
& 0=\alpha \sigma+\beta+\gamma \sigma a_{1}, \\
& 0=\alpha \sigma+\beta \sigma+\gamma\left(1+h \sigma+\left(a_{1}-1-h\right) \sigma_{2}\right),
\end{aligned}
$$

where $h$ is the number of neighbours of $u$ in $D_{1}^{1}$. Observe $\sigma \neq 1$, since $\theta \neq k$. Suppose $\theta=-k$, i.e., $\sigma=-1$. Then from (14), (15) we find $\gamma=0$ and $\alpha=\beta$, which does not work for (16). Thus we have $\sigma^{2} \neq 1$, so we obtain the desired result by solving (14), (15) for $\alpha$ and $\beta$.

\section{Combinatorial regularity and tight edges}

Let $\Gamma$ be a distance-regular graph with diameter $d \geq 2$, eigenvalues $\theta_{0}>\cdots>\theta_{d}$ and $a_{1} \neq 0$. Let $\theta$ be a real number. Suppose an edge $x y$ is tight with respect to $\theta$. Our first result in this section is that local graphs of vertices $x$ and $y$ contain certain combinatorial regularities that we express by $a_{1}$ and the pseudo cosine sequence of $\theta$. Then we show an edge $x y$ is tight with respect to at most two real numbers. We assume the edge $x y$ is tight with respect to real numbers $\theta$ and $\tau$, with $\tau<\theta$, and show $a_{1}-k<\tau \leq \theta_{d}$ and $\theta_{1} \leq \theta$. Finally, we use the first result of this section to derive a connection between the pseudo cosine sequences of $\theta$ and $\tau$ that will later allow us to parameterize the intersection numbers of $\Gamma$ using only $d+1$ parameters.

Before we state our first result we need to determine for which integers $i$ and $j$ the sets $D_{i}^{j}=D_{i}^{j}(x, y)$ are nonempty. Since $\left|D_{i}^{j}\right|=p_{i j}^{1}$, the triangle inequality implies $|i-j| \leq 1$ if $D_{i}^{j} \neq \emptyset$. By Brouwer et al. [2, Prop. 5.5.1], the assumption $a_{1} \neq 0$ implies $a_{i} \neq 0$ for all $i \in\{1, \ldots, d-1\}$. Observe that for all $i \in\{1, \ldots, d\}$ we have

$$
\begin{aligned}
& \left|D_{i-1}^{i}\right|=\left|D_{i}^{i-1}\right|=\frac{b_{1} b_{2} \ldots b_{i-1}}{c_{1} c_{2} \ldots c_{i-1}}, \\
& \left|D_{i}^{i}\right|=\left|\Gamma_{i}(y)\right|-\left|D_{i-1}^{i}\right|-\left|D_{i+1}^{i}\right|=a_{i} \frac{b_{1} b_{2} \ldots b_{i-1}}{c_{1} c_{2} \ldots c_{i}} .
\end{aligned}
$$


Therefore, $D_{i+1}^{i} \neq \emptyset \neq D_{i}^{i+1}$ for all $i \in\{0, \ldots, d-1\}$, and $D_{i}^{i} \neq \emptyset$ for all $i \in$ $\{1, \ldots, d-1\}$. Moreover, $D_{d}^{d}=\emptyset$ if and only if $a_{d}=0$.

Theorem 5.1 Let $\Gamma$ be a distance-regular graph with diameter $d \geq 2$ and $a_{1} \neq 0$. Let $\theta$ be a real number, $\sigma_{0}, \ldots, \sigma_{d}$ the corresponding pseudo cosine sequence. Let us assume an edge $x y$ is tight with respect to $\theta$ and let us set $D_{i}^{j}=D_{i}^{j}(x, y)$. Then the following holds.

(i) For $1 \leq i \leq d$ we have $\sigma_{i-1} \neq \sigma_{i}$ and for all $u \in D_{i-1}^{i}$,

$$
\begin{gathered}
\left|\Gamma_{i-1}(u) \cap D_{1}^{1}\right|=\frac{a_{1}}{1+\sigma} \frac{\sigma \sigma_{i-1}-\sigma_{i}}{\sigma_{i-1}-\sigma_{i}}, \\
\left|\Gamma_{i}(u) \cap D_{1}^{1}\right|=\frac{a_{1}}{1+\sigma} \frac{\sigma_{i-1}-\sigma \sigma_{i}}{\sigma_{i-1}-\sigma_{i}} .
\end{gathered}
$$

(ii) For $1 \leq i \leq d-1$, and for all $v \in D_{i}^{i}$,

$$
\begin{aligned}
\left|\Gamma_{i+1}(v) \cap D_{1}^{1}\right|= & \left|\Gamma_{i-1}(v) \cap D_{1}^{1}\right| \frac{\sigma_{i-1}-\sigma_{i}}{\sigma_{i}-\sigma_{i+1}}+a_{1} \frac{1-\sigma}{1+\sigma} \frac{\sigma_{i}}{\sigma_{i}-\sigma_{i+1}}, \\
\left|\Gamma_{i}(v) \cap D_{1}^{1}\right|= & -\left|\Gamma_{i-1}(v) \cap D_{1}^{1}\right| \frac{\sigma_{i-1}-\sigma_{i+1}}{\sigma_{i}-\sigma_{i+1}}+a_{1} \frac{2 \sigma}{1+\sigma} \\
& -a_{1} \frac{1-\sigma}{1+\sigma} \frac{\sigma_{i+1}}{\sigma_{i}-\sigma_{i+1}}
\end{aligned}
$$

The denominators in (18)-(20) are nonzero.

Proof (i) Observe $D_{1}^{1}$ contains $a_{1}$ vertices, and each is either at distance $i-1$ or $i$ from $u$, so

$$
\left|\Gamma_{i-1}(u) \cap D_{1}^{1}\right|+\left|\Gamma_{i}(u) \cap D_{1}^{1}\right|=a_{1} .
$$

Let $E$ be a pseudo primitive idempotent corresponding to $\theta$. Then we have $E w-$ $a_{1} \sigma(E \hat{x}+E \hat{y}) /(1+\sigma) \in E_{d d}^{*} V$ by Lemma 4.2. Evaluating the $u$ coordinate we obtain

$$
\sigma_{i-1}\left|\Gamma_{i-1}(u) \cap D_{1}^{1}\right|+\sigma_{i}\left|\Gamma_{i}(u) \cap D_{1}^{1}\right|=\frac{a_{1} \sigma}{1+\sigma}\left(\sigma_{i-1}+\sigma_{i}\right) .
$$

Before we proceed, we verify $\sigma_{i-1} \neq \sigma_{i}$. Suppose $\sigma_{i-1}=\sigma_{i}$. Combining (22) and (21), we find $\sigma_{i} a_{1}(1-\sigma)$ is zero. Observe $\sigma_{i} \neq 0$; otherwise $\sigma_{i}=\sigma_{i-1}=0$ and recursively $\sigma_{0}=0$, a contradiction. Observe $\sigma \neq 1$, since $\theta \neq k$. Finally, by assumption $a_{1} \neq 0$, we conclude $\sigma_{i-1} \neq \sigma_{i}$. Solving the system (21), (22), we routinely obtain (18).

(ii) Proceeding as in the proof of (i) part we find

$$
\begin{gathered}
\left|\Gamma_{i-1}(v) \cap D_{1}^{1}\right|+\left|\Gamma_{i}(v) \cap D_{1}^{1}\right|+\left|\Gamma_{i+1}(v) \cap D_{1}^{1}\right|=a_{1}, \\
\sigma_{i-1}\left|\Gamma_{i-1}(v) \cap D_{1}^{1}\right|+\sigma_{i}\left|\Gamma_{i}(v) \cap D_{1}^{1}\right|+\sigma_{i+1}\left|\Gamma_{i+1}(v) \cap D_{1}^{1}\right|=\frac{2 \sigma \sigma_{i} a_{1}}{1+\sigma} .
\end{gathered}
$$


Solving (23), (24) for $\left|\Gamma_{i}(v) \cap D_{1}^{1}\right|,\left|\Gamma_{i+1}(v) \cap D_{1}^{1}\right|$, we routinely obtain (19) and (20).

Corollary 5.2 Let $\Gamma$ be a distance-regular graph with diameter $d \geq 2$ and $a_{1} \neq 0$. Let $\theta$ be a real number and let us assume an edge $x y$ is tight with respect to $\theta$. Then the partition $\left\{\{x\}, D_{1}^{1}(x, y), D_{2}^{1}(x, y)\right\}$ of the local graph of $y$ is equitable, i.e., the subgraphs induced on $D_{1}^{1}(x, y)$ and $D_{2}^{1}(x, y)$ are regular; denoting the valency of the complements resp. by $f$ and $g$, we have

$$
\begin{gathered}
(k+\theta)(1+\theta) f=b_{1}\left(k+\theta\left(a_{1}+1\right)\right), \\
(k+\theta)(1+\theta) g=a_{1}\left(k+\theta\left(a_{1}+1\right)\right) .
\end{gathered}
$$

Moreover, $\theta \neq-1$ and $a_{1} \theta /(k+\theta),-1-b_{1} /(\theta+1)$ are eigenvalues of the local graph of $y$ and are therefore algebraic integers.

Proof For $z \in D_{1}^{1}(x, y)$ define $f(z)=\left|\Gamma_{2}(z) \cap D_{1}^{1}(x, y)\right|$. Setting $i=1$ in (19) we find

$$
f(z)=\frac{1-\sigma}{\sigma-\sigma_{2}}+a_{1} \frac{1-\sigma}{1+\sigma} \frac{\sigma}{\sigma-\sigma_{2}} .
$$

Therefore, $f(z)$ is independent of $z$, and thus the subgraph induced on $D_{1}^{1}(x, y)$ is regular. Evaluate (27) using (7) we get (25). Similarly, by setting $i=2$ in the left equality in (18), we obtain (26), so the subgraph induced on $D_{2}^{1}(x, y)$ is regular. Therefore, the partition $\{x\} \cup D_{1}^{1}(x, y) \cup D_{2}^{1}(x, y)$ is equitable. Observe $\theta \neq-1$, otherwise the LHS of (25) is 0 and the RHS of (25) is $b_{1}^{2}$. By Lemma 4.2, we have $\theta \neq-k$. The eigenvalues of this partition are $a_{1}, a_{1} \theta /(k+\theta),-1-b_{1} /(\theta+1)$. These scalars must be eigenvalues of the local graph of $y$, so they are algebraic integers.

Suppose the assumptions of Corollary 5.2 hold. Then the subgraphs induced on $D_{1}^{1}(x, y)$ and $D_{2}^{1}(x, y)$ are regular, so we can consider the identities in Corollary 5.2 from a different point of view, namely as equations for $\theta$. Since we can obtain the relation $g b_{1}=f a_{1}$ by a two way counting of edges between $D_{1}^{1}(x, y)$ and $D_{2}^{1}(x, y)$, the relations (25) and (26) are equivalent when considered as equations for $\theta$.

Lemma 5.3 Let $\Gamma$ be a distance-regular graph with diameter $d \geq 2$, eigenvalues $\theta_{0}>\cdots>\theta_{d}$ and $a_{1} \neq 0$. Let $x y$ be an edge of $\Gamma$ and $f$ denote the average valency of the complement of the subgraph induced on $D_{1}^{1}(x, y)$. Let $q(\lambda)$ be the following polynomial

$$
(k+\lambda)(1+\lambda) f-b_{1}\left(k+\lambda\left(a_{1}+1\right)\right) .
$$

Then the following (i) and (ii) hold.

(i) Suppose $f=0$. Then $-k /\left(a_{1}+1\right)$ is the only root of $q(\lambda)$.

(ii) Suppose $f \neq 0$. Then the polynomial $q(\lambda)$ has two distinct real roots. The smaller root lies in interval $\left[a_{1}-k, \theta_{d}\right]$ and the bigger root is at least $\theta_{1}$. 
Proof (i) is clear. (ii) Observe, the coefficient of $\lambda^{2}$ in $q(\lambda)$ is $f$. The inequality (11) implies $q\left(\theta_{1}\right) \leq 0$ and $q\left(\theta_{d}\right) \leq 0$. Therefore, the roots $\theta$ and $\tau, \theta \geq \tau$, of the quadratic function $q(\lambda)$ satisfy $\theta \geq \theta_{1}$ and $\tau \leq \theta_{d}$. By (11) and $f \leq b_{1}$, we have $q\left(a_{1}-k\right) \geq 0$, so $a_{1}-k \leq \tau$.

Corollary 5.4 Let $\Gamma$ be a distance-regular graph with diameter $d \geq 2$, eigenvalues $\theta_{0}>\cdots>\theta_{d}$ and $a_{1} \neq 0$. Let $x y$ be an edge of $\Gamma$ and $f$ denote the average valency of the complement of the subgraph induced on $D_{1}^{1}(x, y)$. Then the following (i)-(iv) hold.

(i) For $\theta \in \mathbb{R}$, if the edge $x y$ is tight with respect to $\theta$, then $\theta$ is a root of the polynomial $q(\lambda)$ defined in (28).

(ii) Suppose $f=0$. Then the edge $x y$ is tight with respect to at most one real number.

(iii) Suppose $f \neq 0$. Then the edge $x y$ is tight with respect to at most two distinct real numbers.

(iv) Suppose $\theta, \tau \in \mathbb{R}, \theta>\tau$. If the edge $x y$ is tight with respect to $\theta$ and $\tau$, then $a_{1}-k \leq \tau \leq \theta_{d}$ and $\theta_{1} \leq \theta$.

Theorem 5.5 Let $\Gamma$ be a distance-regular graph with diameter $d \geq 2$ and $a_{1} \neq 0$. Let $\theta$ and $\tau$ be two distinct real numbers, and let $\sigma_{0}, \ldots, \sigma_{d}$ and $\rho_{0}, \ldots, \rho_{d}$ be the corresponding pseudo cosine sequences. Let us assume an edge $x y$ is tight with respect to both $\theta$ and $\tau$. Then

$$
\frac{\sigma \sigma_{i-1}-\sigma_{i}}{(1+\sigma)\left(\sigma_{i-1}-\sigma_{i}\right)}=\frac{\rho \rho_{i-1}-\rho_{i}}{(1+\rho)\left(\rho_{i-1}-\rho_{i}\right)} \quad \text { for all } i \in\{1, \ldots, d\},
$$

and the denominators in (29) are nonzero.

Proof Straightforward by the second equation in (18). The denominators in (29) are nonzero by Lemma 3.1 and Corollary 5.4(iv).

\section{The auxiliary parameter}

Lemma 6.1 Let $\Gamma$ be a distance-regular graph with diameter $d \geq 2$ and $a_{1} \neq 0$. Let $\theta$ and $\tau$ be real numbers such that $\theta>\tau$, and let $\sigma_{0}, \ldots, \sigma_{d}$ and $\rho_{0}, \ldots, \rho_{d}$ respectively be their pseudo cosine sequences. Let us assume an edge $x y$ is tight with respect to both $\theta$ and $\tau$. Then there exists a real scalar $\varepsilon$ such that

$$
\sigma_{i} \rho_{i}-\sigma_{i-1} \rho_{i-1}=\varepsilon\left(\sigma_{i-1} \rho_{i}-\rho_{i-1} \sigma_{i}\right) \quad \text { for all } i \in\{1, \ldots, d\}
$$

Moreover, for each integer $i \in\{1, \ldots, d\}$ the following hold:

(i) $\sigma_{i-1} \neq \varepsilon \sigma_{i}, \quad$ (ii) $\sigma_{i} \neq \varepsilon \sigma_{i-1}$.

Proof Clearing the denominators in (29) and simplifying it using $\varepsilon=(1-\sigma \rho) /(\sigma-$ $\rho$ ), we get (30). By Corollary 5.4(iv), we have $\tau \leq \theta_{d}$ and $\theta_{1} \leq \theta$. Together with 
Lemma 3.1 this implies $\sigma_{i-1} \neq \sigma_{i+1}$ for all $i \in\{1, \ldots, d-1\}$ and $\rho_{i} \neq 0$ for all $i \in\{0, \ldots, d\}$. By $k \neq \theta>\tau$ and $a_{1}-k \leq \tau \leq \theta_{d}$, we have

$$
\begin{aligned}
& \varepsilon+1=(k-\tau)(k+\theta)(\theta-\tau)^{-1} k^{-1}>0 \text { and } \\
& \varepsilon-1=(k+\tau)(k-\theta)(\theta-\tau)^{-1} k^{-1} \neq 0 .
\end{aligned}
$$

Suppose $\sigma_{i-1}=\varepsilon \sigma_{i}$ for some $i \in\{1, \ldots, d\}$. Then, by (30), we find $\sigma_{i} \rho_{i}\left(1-\varepsilon^{2}\right)=0$. Suppose for the moment that $\sigma_{i}=0$. Then $\sigma_{i-1}=0$, contradicting $\sigma_{0}=1$ by (6). Thus $\sigma_{i} \neq 0$. But then we have $\rho_{i}=0$, which is not possible. Hence $\sigma_{i-1} \neq \varepsilon \sigma_{i}$ and (i) holds. The proof of (ii) is similar to the proof of (i).

We refer to $\varepsilon$ as the auxiliary parameter for $\theta$ and $\tau$, and note that $\varepsilon=\left(k^{2}-\right.$ $\theta \tau) /(k(\theta-\tau))$, cf. [7, Sect.8].

Theorem 6.2 Let $\Gamma$ be a distance-regular graph with diameter $d \geq 2$ and $a_{1} \neq 0$. Let $\theta$ and $\tau$ be real numbers such that $\theta>\tau$, and let $\sigma_{0}, \ldots, \sigma_{d}$ and $\rho_{0}, \ldots, \rho_{d}$ respectively be their pseudo cosine sequences. Let us assume an edge xy is tight with respect to both $\theta$ and $\tau$, and let $\varepsilon$ be the auxiliary parameter for $\theta$ and $\tau$. Then

$$
\rho_{i}=\prod_{j=1}^{i} \frac{\sigma_{j-1}-\varepsilon \sigma_{j}}{\sigma_{j}-\varepsilon \sigma_{j-1}} \quad \text { for all } i \in\{1, \ldots, d\}
$$

and the denominators in (31) are nonzero.

Proof By assumptions the relation (30) holds. Rearranging terms in it, we obtain

$$
\rho_{i}\left(\sigma_{i}-\varepsilon \sigma_{i-1}\right)=\rho_{i-1}\left(\sigma_{i-1}-\varepsilon \sigma_{i}\right) \text { for all } i \in\{1, \ldots, d\} .
$$

Observe $\sigma_{i} \neq \varepsilon \sigma_{i-1}$ for $1 \leq i \leq d$ by Lemma 6.1(ii), so the coefficient of $\rho_{i}$ in (32) is never zero. Solving that equation for $\rho_{i}$ and applying induction, we routinely obtain (31).

\section{A parameterization}

We begin with a result about arbitrary distance-regular graphs.

Lemma 7.1 Let $\Gamma$ be a distance-regular graph with diameter $d \geq 2$, eigenvalues $\theta_{0}>\cdots>\theta_{d}$ and $a_{1} \neq 0$. Let $\theta$ and $\tau$ be distinct real numbers, such that $k \neq \theta \geq \theta_{1}$ and $\tau \leq \theta_{d}$. Let $\sigma_{0}, \ldots, \sigma_{d}$ and $\rho_{0}, \ldots, \rho_{d}$ denote the corresponding pseudo cosine sequences. Then the intersection numbers of $\Gamma$ are given by

$$
\begin{aligned}
k & =\frac{\left(\sigma-\sigma_{2}\right)(1-\rho)-\left(\rho-\rho_{2}\right)(1-\sigma)}{\left(\rho-\rho_{2}\right)(1-\sigma) \sigma-\left(\sigma-\sigma_{2}\right)(1-\rho) \rho}, \\
b_{i} & =k \frac{\left(\sigma_{i-1}-\sigma_{i}\right)(1-\rho) \rho_{i}-\left(\rho_{i-1}-\rho_{i}\right)(1-\sigma) \sigma_{i}}{\left(\rho_{i}-\rho_{i+1}\right)\left(\sigma_{i-1}-\sigma_{i}\right)-\left(\sigma_{i}-\sigma_{i+1}\right)\left(\rho_{i-1}-\rho_{i}\right)},
\end{aligned}
$$




$$
c_{i}=k \frac{-\left(\sigma_{i+1}-\sigma_{i}\right)(1-\rho) \rho_{i}+\left(\rho_{i+1}-\rho_{i}\right)(1-\sigma) \sigma_{i}}{\left(\rho_{i}-\rho_{i+1}\right)\left(\sigma_{i-1}-\sigma_{i}\right)-\left(\sigma_{i}-\sigma_{i+1}\right)\left(\rho_{i-1}-\rho_{i}\right)},
$$

for $i \in\{1, \ldots, d-1\}$, and the denominators in (33)-(35) are nonzero.

Proof To obtain (34) and (35) pick any integer $i(1 \leq i \leq d-1)$, and recall, by (7), that

$$
\begin{aligned}
& c_{i}\left(\sigma_{i-1}-\sigma_{i}\right)-b_{i}\left(\sigma_{i}-\sigma_{i+1}\right)=k(\sigma-1) \sigma_{i}, \\
& c_{i}\left(\rho_{i-1}-\rho_{i}\right)-b_{i}\left(\rho_{i}-\rho_{i+1}\right)=k(\rho-1) \rho_{i} .
\end{aligned}
$$

To solve this linear system for $c_{i}$ and $b_{i}$, consider the determinant

$$
\begin{aligned}
D_{i} & :=\operatorname{det}\left(\begin{array}{cc}
\sigma_{i-1}-\sigma_{i} & \sigma_{i}-\sigma_{i+1} \\
\rho_{i-1}-\rho_{i} & \rho_{i}-\rho_{i+1}
\end{array}\right) \\
& =\left(\rho_{i}-\rho_{i+1}\right)\left(\sigma_{i-1}-\sigma_{i}\right)-\left(\sigma_{i}-\sigma_{i+1}\right)\left(\rho_{i-1}-\rho_{i}\right) .
\end{aligned}
$$

By Lemma 3.1, we have $\rho_{i}-\rho_{i+1} \neq 0 \neq \rho_{i}-\rho_{i-1}, \sigma_{i-1}-\sigma_{i} \neq 0 \neq \sigma_{i}-\sigma_{i+1}$, and $\operatorname{sgn}\left(\rho_{\mathrm{i}}-\rho_{\mathrm{i}+1}\right)=\operatorname{sgn}\left(\rho_{\mathrm{i}}-\rho_{\mathrm{i}-1}\right), \operatorname{sgn}\left(\sigma_{\mathrm{i}-1}-\sigma_{\mathrm{i}}\right)=\operatorname{sgn}\left(\sigma_{\mathrm{i}}-\sigma_{\mathrm{i}+1}\right)$, thus $D_{i} \neq 0$. Now equations (36), (37) has the unique solution (34), (35) by elementary linear algebra. The denominators in (34) and (35) equal $D_{i}$; in particular they are not zero. To get (33), we use (35) and the fact that $c_{1}=1$.

Theorem 7.2 Let $\Gamma$ be a distance-regular graph with diameter $d \geq 2$ and $a_{1} \neq 0$. Let $\theta$ and $\tau$ be real numbers such that $\theta>\tau$, and let $\sigma_{0}, \ldots, \sigma_{d}$ be the pseudo cosine sequence corresponding to $\theta$. Let us assume an edge $x y$ is tight with respect to both $\theta$ and $\tau$. Let $\varepsilon$ denote the auxiliary parameter for $\theta$ and $\tau$. Then the intersection numbers are given by

$$
\begin{aligned}
k & =h \frac{\sigma-\varepsilon}{\sigma-1}, \\
b_{i} & =h \frac{\left(\sigma_{i-1}-\sigma \sigma_{i}\right)\left(\sigma_{i+1}-\varepsilon \sigma_{i}\right)}{\left(\sigma_{i-1}-\sigma_{i+1}\right)\left(\sigma_{i+1}-\sigma_{i}\right)}, \\
c_{i} & =-h \frac{\left(\sigma_{i+1}-\sigma \sigma_{i}\right)\left(\sigma_{i-1}-\varepsilon \sigma_{i}\right)}{\left(\sigma_{i-1}-\sigma_{i+1}\right)\left(\sigma_{i-1}-\sigma_{i}\right)},
\end{aligned}
$$

for $i \in\{1, \ldots, d-1\}$, where

$$
h=\frac{(1-\sigma)\left(1-\sigma_{2}\right)}{\left(\sigma^{2}-\sigma_{2}\right)(1-\varepsilon \sigma)} .
$$

We remark the denominators in (38)-(41) are all nonzero.

Proof Eliminating the pseudo cosine sequence $\rho_{0}, \ldots, \rho_{d}$ corresponding to $\tau$ in (33)-(35) using (31), we routinely obtain (38)-(41). The denominator of $h$ is nonzero by Corollary 5.4(ii), Theorem 5.1(i) and Lemma 6.1(ii). The denominators in (38)-(39) are nonzero by Lemma 3.1 and Corollary 5.4(iv). 
Corollary 7.3 Let $\Gamma$ be a distance-regular graph with diameter $d \geq 2$ and $a_{1} \neq 0$. Let $\theta$ and $\tau$ be real numbers such that $\theta>\tau$, and let $\sigma_{0}, \ldots, \sigma_{d}$ be the pseudo cosine sequences corresponding to $\theta$. Let us assume an edge $x y$ is tight with respect to both $\theta$ and $\tau$. Let $\varepsilon$ denote the auxiliary parameter for $\theta$ and $\tau$. Then

$$
a_{i}=g \frac{\left(\sigma_{i+1}-\sigma \sigma_{i}\right)\left(\sigma_{i-1}-\sigma \sigma_{i}\right)}{\left(\sigma_{i+1}-\sigma_{i}\right)\left(\sigma_{i-1}-\sigma_{i}\right)},
$$

for $i \in\{1, \ldots, d\}$, where

$$
g=\frac{(\varepsilon-1)\left(1-\sigma_{2}\right)}{\left(\sigma^{2}-\sigma_{2}\right)(1-\varepsilon \sigma)} .
$$

We remark the denominators in (42)-(43) are all nonzero.

Proof Evaluate the right hand side of $a_{i}=k-b_{i}-c_{i}$ using (38)-(41), and simplify.

\section{The pseudo 1-homogeneous property}

In this section we generalize the 1-homogeneous property to the pseudo 1-homogeneous property.

Let $\Gamma$ be a distance-regular graph with diameter $d$ and let $x y$ be an edge of $\Gamma$. Set $D_{i}^{j}=D_{i}^{j}(x, y)$. Then we say $\Gamma$ is pseudo 1 -homogeneous with respect to $x y$ whenever the partition

$$
\left\{D_{i}^{j} \mid i, j=0, \ldots, d, \text { and }(i, j) \neq(d, d)\right\}
$$

is equitable in the subgraph induced on $\cup_{(i j) \neq(d d)} D_{i j}$. Let $\theta$ and $\tau$ be real numbers such that $\theta>\tau$. We assume $d \geq 2, a_{1} \neq 0$ and the edge $x y$ is tight with respect to both $\theta$ and $\tau$. We show that $\Gamma$ is pseudo 1-homogeneous with respect to $x y$. We do this by calculating all the parameters that correspond to the partition (44) in terms of the intersection array of $\Gamma$ and the pseudo cosine sequence corresponding to $\theta$.

Theorem 8.1 Let $\Gamma$ be a distance-regular graph with diameter $d \geq 2$ and $a_{1} \neq 0$. Let $\theta$ and $\tau$ be real numbers such that $\theta>\tau$. Let $\sigma_{0}, \ldots, \sigma_{d}$ be the pseudo cosine sequence corresponding to $\theta$. Let $x y$ be an edge of $\Gamma$. Suppose $x y$ is tight with respect to both $\theta$ and $\tau$. Then for all integers $i \in\{1, \ldots, d-1\}$, and for all vertices $v \in$ $D_{i}^{i}(x, y)$,

$$
\begin{aligned}
& \left|\Gamma_{i-1}(v) \cap D_{1}^{1}(x, y)\right|=c_{i} \frac{\left(\sigma^{2}-\sigma_{2}\right)\left(\sigma_{i}-\sigma_{i+1}\right)}{\left(\sigma-\sigma_{2}\right)\left(\sigma \sigma_{i}-\sigma_{i+1}\right)}, \\
& \left|\Gamma_{i+1}(v) \cap D_{1}^{1}(x, y)\right|=b_{i} \frac{\left(\sigma^{2}-\sigma_{2}\right)\left(\sigma_{i-1}-\sigma_{i}\right)}{\left(\sigma-\sigma_{2}\right)\left(\sigma_{i-1}-\sigma \sigma_{i}\right)} .
\end{aligned}
$$

Moreover, the denominators in (45), (46) and numbers $\left|\Gamma_{i-1}(v) \cap D_{1}^{1}(x, y)\right|$, $\left|\Gamma_{i+1}(v) \cap D_{1}^{1}(x, y)\right|$ are nonzero. 
Proof Let $\rho_{0}, \ldots, \rho_{d}$ denote pseudo cosine sequence for $\tau$ and set $D_{i}^{j}=D_{i}^{j}(x, y)$. The edge $x y$ is tight with respect to both $\theta$ and $\tau$, so we have, by Theorem 5.1,

$$
\begin{aligned}
& \left|\Gamma_{i+1}(v) \cap D_{1}^{1}\right|=\left|\Gamma_{i-1}(v) \cap D_{1}^{1}\right| \frac{\sigma_{i-1}-\sigma_{i}}{\sigma_{i}-\sigma_{i+1}}+a_{1} \frac{1-\sigma}{1+\sigma} \frac{\sigma_{i}}{\sigma_{i}-\sigma_{i+1}}, \\
& \left|\Gamma_{i+1}(v) \cap D_{1}^{1}\right|=\left|\Gamma_{i-1}(v) \cap D_{1}^{1}\right| \frac{\rho_{i-1}-\rho_{i}}{\rho_{i}-\rho_{i+1}}+a_{1} \frac{1-\rho}{1+\rho} \frac{\rho_{i}}{\rho_{i}-\rho_{i+1}} .
\end{aligned}
$$

Eliminating $\rho_{0}, \ldots, \rho_{d}$ in (48) using (31), we obtain

$$
\begin{aligned}
\left|\Gamma_{i+1}(v) \cap D_{1}^{1}\right|= & \left|\Gamma_{i-1}(v) \cap D_{1}^{1}\right| \frac{\sigma_{i-1}-\sigma_{i}}{\sigma_{i}-\sigma_{i+1}} \frac{\sigma_{i+1}-\varepsilon \sigma_{i}}{\sigma_{i-1}-\varepsilon \sigma_{i}} \\
& +a_{1} \frac{(1-\sigma)\left(\sigma_{i+1}-\varepsilon \sigma_{i}\right)}{(1+\sigma)(1-\varepsilon)\left(\sigma_{i}-\sigma_{i+1}\right)}
\end{aligned}
$$

where $\varepsilon$ denotes the auxiliary parameter for $\theta$ and $\tau$. Solving (47), (49) for $\mid \Gamma_{i+1}(v) \cap$ $D_{1}^{1}(x, y) \mid$ and $\left|\Gamma_{i-1}(v) \cap D_{1}^{1}(x, y)\right|$, and evaluating the result using (39)-(43), we get (45), (46) as desired. The denominators in (45), (46) and the numbers $\mid \Gamma_{i-1}(v) \cap$ $D_{1}^{1}(x, y)|,| \Gamma_{i+1}(v) \cap D_{1}^{1}(x, y) \mid$ are nonzero by Theorem 7.2.

Theorem 8.2 Let $\Gamma$ be a distance-regular graph with diameter $d \geq 2$, and $a_{1} \neq 0$. Let $\theta$ and $\tau$ be real numbers such that $\theta>\tau$, and let $\sigma_{0}, \ldots, \sigma_{d}$ be the pseudo cosine sequences corresponding to $\theta$. Let $x y$ be an edge of $\Gamma$. Suppose $x y$ is tight with respect to both $\theta$ and $\tau$. Then the following (i), (ii) hold.

(i) For all integers $i(1 \leq i \leq d-1)$, and for all $v \in D_{i}^{i}(x, y)$,

$$
\begin{aligned}
& \left|\Gamma(v) \cap D_{i-1}^{i-1}(x, y)\right|=c_{i} \frac{\left(\sigma_{i}-\sigma_{i+1}\right)\left(\sigma \sigma_{i-1}-\sigma_{i}\right)}{\left(\sigma_{i-1}-\sigma_{i}\right)\left(\sigma \sigma_{i}-\sigma_{i+1}\right)}, \\
& \left|\Gamma(v) \cap D_{i+1}^{i+1}(x, y)\right|=b_{i} \frac{\left(\sigma_{i-1}-\sigma_{i}\right)\left(\sigma_{i}-\sigma \sigma_{i+1}\right)}{\left(\sigma_{i}-\sigma_{i+1}\right)\left(\sigma_{i-1}-\sigma \sigma_{i}\right)} .
\end{aligned}
$$

(ii) For all integers $i(2 \leq i \leq d)$, and for all $u \in D_{i-1}^{i}(x, y) \cup D_{i}^{i-1}(x, y)$,

$$
\left|\Gamma(u) \cap D_{i-1}^{i-1}(x, y)\right|=a_{i-1} \frac{(1-\sigma)\left(\sigma_{i-1}^{2}-\sigma_{i-2} \sigma_{i}\right)}{\left(\sigma_{i-1}-\sigma_{i}\right)\left(\sigma_{i-2}-\sigma \sigma_{i-1}\right)} .
$$

Proof (i) To prove (50), we assume $i \geq 2$; otherwise both sides are zero. Let $\alpha_{i}$ denote the expression on the left side of (45). Let us fix $v \in D_{i}^{i}(x, y)$. Let $N$ be the number of ordered pairs $p q$ such that

$$
p \in \Gamma_{i-1}(v) \cap D_{1}^{1}(x, y), \quad q \in \Gamma(v) \cap D_{i-1}^{i-1}(x, y), \quad \partial(p, q)=i-2 .
$$

We compute $N$ in two ways. On one hand, there are precisely $\alpha_{i}$ choices for $p$, and given $p$, there are precisely $c_{i-1}$ choices for $q$, so $N=\alpha_{i} c_{i-1}$. On the other hand, there are precisely $\left|\Gamma(v) \cap D_{i-1}^{i-1}(x, y)\right|$ choices for $q$, and given $q$, there are precisely $\alpha_{i-1}$ choices for $p$, hence $N=\left|\Gamma(v) \cap D_{i-1}^{i-1}(x, y)\right| \alpha_{i-1}$. By Theorem 8.1, 
we have $\alpha_{i-1} \neq 0$; combining this with the obtained expressions for $N$, we find $\left|\Gamma(v) \cap D_{i-1}^{i-1}(x, y)\right|=c_{i-1} \alpha_{i} / \alpha_{i-1}$. Eliminating $\alpha_{i-1}$ and $\alpha_{i}$ using (45), we obtain (50), as desired.

We now show (51). Let us first assume $i \leq d-2$. Let $\beta_{i}$ denote the expression on the left side of (46). Let $N^{\prime}$ be the number of ordered pairs $p q$ such that

$$
p \in \Gamma_{i+1}(v) \cap D_{1}^{1}(x, y), \quad q \in \Gamma(v) \cap D_{i+1}^{i+1}(x, y), \quad \partial(p, q)=i+2 .
$$

We compute $N^{\prime}$ in two ways. On one hand, there are precisely $\beta_{i}$ choices for $p$, and given $p$, there are precisely $b_{i+1}$ choices for $q$. Since $x$ and $y$ lie on a geodesic between $p$ and $v$, these choices for $q$ lie in $D_{i+1}^{i+1}(x, y)$ as well. Therefore, $N^{\prime}=$ $\beta_{i} b_{i+1}$. On the other hand, there are precisely $\left|\Gamma(v) \cap D_{i+1}^{i+1}(x, y)\right|$ choices for $q$, and given $q$, there are precisely $\beta_{i+1}$ choices for $p$, hence $N^{\prime}=\left|\Gamma(v) \cap D_{i+1}^{i+1}(x, y)\right| \beta_{i+1}$. By Theorem 8.1, we have $\beta_{i+1} \neq 0$; combining this with the obtained expressions for $N^{\prime}$, we find $\left|\Gamma(v) \cap D_{i+1}^{i+1}(x, y)\right|=b_{i+1} \beta_{i} / \beta_{i+1}$. Eliminating $\beta_{i}$ and $\beta_{i+1}$ using (46), we obtain (51), as desired.

(ii) Let $\gamma_{i}$ denote the expressions on the left side of the left identity in (18). By a two way counting of pairs of vertices $(p, q)$ such that $p \in \Gamma_{i-1}(u) \cap D_{1}^{1}(x, y)$ and $q \in \Gamma(u) \cap \Gamma_{i-2}(p)$, we easily have

$$
\gamma_{i} c_{i-1}=\left|\Gamma(u) \cap D_{i-1}^{i-1}(x, y)\right| \alpha_{i-1}+\gamma_{i-1} c_{i-1} .
$$

Since $\alpha_{i-1} \neq 0$ by Theorem 8.1 , we find $\left|\Gamma(u) \cap D_{i-1}^{i-1}(x, y)\right|=c_{i-1}\left(\gamma_{i}-\gamma_{i-1}\right) / \alpha_{i-1}$, by symmetry, the same is true if $u \in D_{i}^{i-1}(x, y)$. Now (52) is calculated by (18) and (45).

(i)-cont. It remains to consider the case when $i=d-1$ in (i). A two way counting of paths of length $d-1$ from $v$ to $D_{1}^{1}(x, y)$ implies that $\left|\Gamma(v) \cap D_{d}^{d}(x, y)\right|$ is independent of $v \in D_{d-1}^{d-1}(x, y)$, cf. [6, Proof of Thm. 3.1]. (51) now follows from a two way counting of edges between $D_{d}^{d-1}(x, y)$ and $D_{d-1}^{d-1}(x, y)$.

\section{Two characterizations}

In this section we prove two characterizations. For, we need the following results about an action of the Bose-Mesner algebra.

Lemma 9.1 Let $\Gamma$ be a distance-regular graph with diameter $d \geq 2$ and $a_{1} \neq 0$. Let $x y$ be an edge $\Gamma$ and let $H=H(x, y)$ as in Section 1 . Suppose $\Gamma$ is pseudo 1-homogeneous with respect to $x y$. Then

$$
\mathcal{M} H \subseteq \operatorname{Span}\left\{w_{i j} \mid i, j=0, \ldots, d \text { and }(i, j) \neq(d, d)\right\}+E_{d d}^{*} V .
$$

Proof For $i<d$ we have $A_{i} \hat{x} \in \operatorname{Span}\left(w_{i, i-1}, w_{i, i}, w_{i, i+1}\right)$. Similarly, taking into account Theorem 5.1(i)-(ii) (which tells us what are possible distances from a vertex to the vertices in $D_{1}^{1}(x, y)$, cf. (21), (23)), we have for $i \leq d-2$

$$
A_{i} w \in \operatorname{Span}\left(w_{i-1, i-1}, w_{i-1, i}, w_{i, i-1}, w_{i, i}, w_{i+1, i}, w_{i, i+1}, w_{i+1, i+1}\right) .
$$


The above inclusion (54) holds also for $i=d-1$ only if we substitute $w_{d d}$ on the RHS by $E_{d d}^{*} V$. Since the matrices $A_{0}, \ldots, A_{d-1}, J$ form a basis for $\mathcal{M}$, it remains to consider the action by $J$. We have $J \hat{x}=j, J \hat{y}=j$, and $J w=a_{1} j$, where $j$ is the all 1 's vector. Observe $j$ is in the RHS of (53), so the relation (53) has been proved.

Theorem 9.2 Let $\Gamma$ be a distance-regular graph with diameter $d \geq 2$ and $a_{1} \neq 0$. Then for every edge $x y$ the following are equivalent.

(i) The graph $\Gamma$ is pseudo 1-homogeneous with respect to the edge $x y$, and the subgraph induced on $D_{1}^{1}(x, y)$ is not a clique.

(ii) The edge $x y$ is tight with respect to two distinct real numbers.

Proof (i) $\Longrightarrow$ (ii): Let $\theta_{0}>\cdots>\theta_{d}$ be the eigenvalues of $\Gamma$. Let $t$ be the number defined by (3). By [7, Def. 5.1 and Cor. 3.6] we have $t \in\{0,1,2\}$. If $t=2$ then $x y$ is tight with respect to $\theta_{1}$ and $\theta_{d}$. It remains to consider the case when $t<2$.

Choose $\theta$ to be one of the roots of the polynomial given by (28), and let $E$ be a pseudo primitive idempotent for $\theta$. Let $\sigma_{0}, \ldots, \sigma_{d}$ be the pseudo cosine sequence corresponding to $E$. Then $\sigma+1 \neq 0$ by Lemma 5.3. Let us define $\Delta=w-\alpha(\hat{x}+\hat{y})$, where $\alpha=a_{1} \sigma /(1+\sigma)$. We show that the edge $x y$ is tight with respect to $\theta$. To do this we first show that $E \Delta \in E_{d d}^{*} V$ and then that $\theta \neq k$.

We start by verifying the vector $E \Delta$ is orthogonal to $\hat{x}, \hat{y}$ and $\hat{z}$ for all $z \in$ $D_{1}^{1}(x, y)$. By Lemma 3.2 and our choice of $\alpha$, the $x$ and $y$ coordinates of $E \Delta$ equal to $a_{1} \sigma-\alpha(1+\sigma)=0$. Now let $z \in D_{1}^{1}(x, y)$ and recall that $\left|\Gamma_{2}(z) \cap D_{1}^{1}(x, y)\right|=f$. Then the $z$ coordinate of $E \Delta$ is, by Lemma 3.2 and our choice of $\theta$, equal to

$$
1+f \sigma_{2}+\left(a_{1}-f-1\right) \sigma-\frac{2 a_{1} \sigma^{2}}{1+\sigma}=\frac{k-\theta}{k+\theta} q(\theta)=0,
$$

where $q(\theta)$ is the polynomial defined in (28). As $E$ is an element of the Bose-Mesner algebra $\mathcal{M}$ of $\Gamma$ and the graph $\Gamma$ is pseudo 1-homogeneous, we have, by Lemma 9.1,

$$
E \Delta-\sum_{i j} \alpha_{i j} w_{i j} \in E_{d d}^{*} V
$$

for some real numbers $\alpha_{i j}$. We need to show that $\alpha_{i j}=0$ for $i \neq d$ or $j \neq d$. We will show for all $s \in\{1, \ldots, d-1\}$ that $\alpha_{s-1, s}=\alpha_{s, s-1}=\alpha_{s s}=0$ by induction on $s$. For $s=1$ we have $\alpha_{10}=\alpha_{01}=\alpha_{11}=0$, since $E \Delta$ is orthogonal to $w_{10}=\hat{x}, w_{01}=\hat{y}$ and $w=w_{11}$. Let us now suppose $s>1$. Then, by induction assumption, $\alpha_{i j}=0$ for $i<s$ and $j<s$. Since $E$ is a pseudo primitive idempotent, we have, by Lemma 3.3, $(A-\theta I) E \in \operatorname{Span}\left(A_{d}\right)$, which implies

$$
(A-\theta I) E \Delta \in \operatorname{Span} A_{d} \Delta \subseteq \operatorname{Span}\left(w_{d-1, d}, w_{d, d-1}, w_{d-1, d-1}\right)+E_{d d}^{*} V .
$$

Now $\alpha_{s-1, s}=0$, since otherwise $(A-\theta I) E \Delta$ has a nonzero contribution from $w_{s-2, s-1}$ contradicting (56). Similarly $\alpha_{s, s-1}=0$. We will now prove $\alpha_{s s}=0$. For, we first show that there exists an edge between $D_{s-1}^{s-1}(x, y)$ and $D_{s}^{s}(x, y)$. Suppose the opposite. By the definition of $H$, we have

$$
\mathcal{M} H=\mathcal{M} \hat{x}+\mathcal{M} \hat{y}+\mathcal{M} w .
$$


The dimension of $\mathcal{M}$ is $d+1$ and $J \hat{x}=J \hat{y}$. We conclude that the dimension of $\mathcal{M} \hat{x}+\mathcal{M} \hat{y}$ is at most $2 d+1$. Because there is no edge between $D_{s-1}^{s-1}(x, y)$ and $D_{s}^{s}(x, y)$, we need at least $s$ steps to get from $D_{1}^{1}(x, y)$ to $D_{s}^{s}(x, y)$ and thus $A_{s-1} w$ lies in the $\operatorname{Span}\left\{A_{0} w, A_{1} w, \ldots, A_{s-2} w\right\}+\mathcal{M} \hat{x}+\mathcal{M} \hat{y}$. Hence the dimension of $\mathcal{M} H$ is at most $2 d+s \leq 3 d-1$. This contradicts (3) with $t<2$.

Now $\alpha_{s s}=0$, since otherwise $(A-\theta I) E \Delta$ has a nonzero contribution from $w_{s-1, s-1}$ contradicting (56). We now have $\alpha_{s-1, s}=\alpha_{s, s}=\alpha_{s, s-1}=0$ for all $s \in$ $\{1, \ldots, d-1\}$. Let us finally show $\alpha_{d-1, d}=\alpha_{d, d-1}=0$. Now $\alpha_{d-1, d}=0$, since otherwise $(A-\theta I) E \Delta$ has a nonzero contribution from $w_{d-2, d-1}$. Similarly $\alpha_{d, d-1}=0$. Therefore, we have $E \Delta \in E_{d d}^{*} V$.

Since $D_{1}^{1}(x, y)$ does not induce a clique, we have $f>0$, and, by Lemma 5.3, there are two distinct roots of the polynomial defined in (28). We can assume that $\theta \neq k$, and so the edge $x y$ is tight with respect to $\theta$. It remains to prove that the other root $\tau$, say, is also not equal to $k$. By Corollary 5.2, $a_{1} \theta /(k+\theta)$ is an algebraic integer. An easy calculation shows that this number equals $-1-b_{1} /(\tau+1)$. If $\tau=k$ then this is a rational algebraic integer and hence integer, so $\tau+1=k+1$ must divide $b_{1}$. But $\tau+1=k+1>b_{1}$, so $\tau \neq k$.

Therefore, the edge $x y$ is tight with respect to two distinct real numbers.

(ii) $\Longrightarrow$ (i): This follows directly from Theorem 8.2 and Corollary 5.4(ii).

Theorem 9.3 Let $\Gamma$ be a distance-regular graph with diameter $d \geq 2$, and let $x$ be a vertex of $\Gamma$, such that the local graph $\Delta(x)$ is connected. Then the following are equivalent.

(i) For all $y \in \Gamma(x)$, the graph $\Gamma$ is pseudo 1-homogeneous with respect to the edge $x y$.

(ii) There exist real numbers $\sigma_{0}, \ldots, \sigma_{d}, \varepsilon, h$ such that $\sigma_{0}=1, \varepsilon \neq-1$,

$$
\begin{aligned}
k & =h \frac{\sigma-\varepsilon}{\sigma-1}, \\
b_{i} & =h \frac{\left(\sigma_{i-1}-\sigma \sigma_{i}\right)\left(\sigma_{i+1}-\varepsilon \sigma_{i}\right)}{\left(\sigma_{i-1}-\sigma_{i+1}\right)\left(\sigma_{i+1}-\sigma_{i}\right)} \quad(1 \leq i \leq d-1), \\
c_{i} & =h \frac{\left(\sigma_{i+1}-\sigma \sigma_{i}\right)\left(\sigma_{i-1}-\varepsilon \sigma_{i}\right)}{\left(\sigma_{i+1}-\sigma_{i-1}\right)\left(\sigma_{i-1}-\sigma_{i}\right)} \quad(1 \leq i \leq d-1),
\end{aligned}
$$

and the local graph of $x$ is strongly regular with nontrivial eigenvalues $a_{1} \sigma /(1+$ $\sigma)$ and $\left(\sigma_{2}-1\right) /\left(\sigma-\sigma_{2}\right)$.

Moreover, suppose ( $i$ ) and (ii) hold. Then $\sigma_{0}, \ldots, \sigma_{d}$ is a pseudo cosine sequence for $k \sigma$. For all $y \in \Gamma(x)$ the edge $x y$ is tight with respect to $k \sigma$ and $k \rho$, where $\rho=$ $(1-\varepsilon \sigma) /(\sigma-\varepsilon)$, the scalar $\varepsilon$ is the auxiliary parameter for $k \sigma$ and $k \rho$, and $h=$ $(1-\sigma)\left(1-\sigma_{2}\right) /\left(\left(\sigma^{2}-\sigma_{2}\right)(1-\varepsilon \sigma)\right)$.

Proof (i) $\Longrightarrow$ (ii): For a neighbour $y$ of $x$ we denote by $f_{y}$ the valency of the complement of the subgraph induced on $D_{1}^{1}(x, y)$. Since the local graph $\Delta(x)$ is connected, 
$D_{1}^{1}(x, y)$ does not induce a clique. By Theorem 9.2, every edge $x y, y \in \Gamma(x)$, is tight with respect to two distinct real numbers that are solutions of the quadratic polynomial

$$
(k+\lambda)(\lambda+1) f_{y}-b_{1}\left(k+\lambda\left(a_{1}+1\right)\right) .
$$

Then for every $y \in \Gamma(x)$ the distance partition of the local graph $\Delta(x)$, i.e., $\left\{\{y\}, D_{1}^{1}(x, y), D_{1}^{2}(x, y)\right\}$, is equitable by Corollary 5.2. Therefore, $\Delta(x)$ is strongly regular by [6, Prop. 2.1] and $f=f_{y}$ is independent of $y$. Let $\theta$ and $\tau$ be the solutions of the above quadratic, so that $\theta>\tau$. Let $\sigma_{0}, \ldots, \sigma_{d}$ be a pseudo cosine sequence for $\theta$. Then, by Theorem 9.2 and Theorem 7.2, we obtain the desired parameterization of entries of intersection array $\left\{b_{0}, \ldots, b_{d-1} ; c_{1}, \ldots, c_{d}\right\}$ and the corresponding denominators are nonzero.

Let $\left(v^{\prime}, k^{\prime}, \lambda^{\prime}, \mu^{\prime}\right)$ be the parameters of the local graph $\Delta(x)$. Then $v^{\prime}=k, k^{\prime}=a_{1}$, $\lambda^{\prime}=a_{1}-1-f, \mu^{\prime}=a_{1} f /\left(k-1-a_{1}\right)$. Furthermore, $a_{1} \sigma /(1+\sigma)$ and $\left(\sigma_{2}-1\right) /(\sigma-$ $\left.\sigma_{2}\right)$ are its eigenvalues by Corollary 5.2.

(ii) $\Longrightarrow$ (i): First we get from $c_{1}=1$ that $h=(1-\sigma)\left(1-\sigma_{2}\right) /\left(\left(\sigma^{2}-\sigma_{2}\right)(1-\varepsilon \sigma)\right)$. Then we verify that for the given $b_{i}$ 's and $c_{i}$ 's the identity (7) is satisfied. Therefore, $\sigma_{0}, \ldots, \sigma_{d}$ is a nontrivial pseudo cosine sequence for $k \sigma$. Let us denote this number by $\theta$. Let $\rho_{0}, \ldots, \rho_{d}$ be defined by $\rho_{0}=1$ and (31). Then $\varepsilon=(1-\sigma \rho) /(\sigma-\rho)$ and the identity (7) is satisfied by $\rho_{i}$ 's in place of $\sigma_{i}$ 's. Therefore, by $\varepsilon \neq-1$, a pseudo cosine sequence $\rho_{0}, \ldots, \rho_{d}$ for $k \rho$ is nontrivial. Let us denote $k \rho$ by $\tau$.

Let $r$ and $s$ denote the nontrivial eigenvalues of the local graph $\Delta(x)$ and let $m_{r}$ and $m_{s}$ be respectively their multiplicities. Thus $r=a_{1} \sigma /(1+\sigma), s=-(1-$ $\left.\sigma_{2}\right) /\left(\sigma-\sigma_{2}\right)$ and, by [2, Thm. 1.3.1], also

$$
m_{r}=\frac{(1+\sigma)(\sigma-\varepsilon)}{\sigma_{2}-\sigma^{2}}, \quad m_{s}=-\frac{(1-\varepsilon)(1+\sigma)\left(\sigma_{2}-\varepsilon \sigma\right)}{\left(\sigma_{2}-\sigma^{2}\right)(1-\varepsilon \sigma)} .
$$

Then the following inequality holds with equality for $i=d-1$ :

$$
1+\frac{p_{i-1}(\tilde{r})}{p_{i}(\tilde{r})(1+\tilde{r})} m_{r}+\frac{p_{i-1}(\tilde{s})}{p_{i}(\tilde{s})(1+\tilde{s})} m_{s} \leq \frac{k}{b_{i}} \quad(1 \leq i \leq d-1),
$$

where $\tilde{r}=-1-b_{1} /(1+r)=\tau$ and $\tilde{s}=-1-b_{1} /(1+s)=\theta$. To verify this claim use the identity for $p_{i}(\theta)$ in (8), the above formulas for numbers of the intersection array of $\Gamma$, and the formulas for the pseudo cosine sequences $\rho_{0}, \ldots, \rho_{d}$ in term of the pseudo cosine sequence $\sigma_{0}, \ldots, \sigma_{d}$ and $\varepsilon$ that we used here to define $\rho_{i}$ 's.

Finally, every irreducible $T$-module with endpoint 1 is thin by [10, Thm. 13.6], and $\Gamma$ is pseudo 1-homogeneous by Curtin and Nomura [3, Thm. 1.6]. Therefore, by Theorem 9.2, the edge $x y$ is tight with respect to two real numbers. But then these two numbers have to be $\theta$ and $\tau$, so $\varepsilon$ is the auxiliary parameter for $\theta$ and $\tau$.

\section{Conclusion}

Let $\Gamma$ be a distance-regular graph with diameter $d \geq 2, a_{1} \neq 0$ and $a_{d} \neq 0$. Suppose $\Gamma$ is 1 -homogeneous with respect to an edge $x y$. By $a_{d} \neq 0$, the graph $\Gamma$ is not tight, 
hence $t=1$ (by $\mathcal{M} H=W$ and (2)). Then we have, by Corollary 5.2,

$$
f(x, y)=b_{1} \frac{k+\theta\left(a_{1}+1\right)}{(k+\theta)(1+\theta)}, \quad \text { for } \theta \in\left\{\theta_{1}, \theta_{d}\right\},
$$

where $f=f(x, y)$ was defined in Section 4. There are examples with $\theta=\theta_{1}$, namely the halved cubes of the cubes of odd diameter and the complement of the HigmanSims graph, and there are also examples with $\theta=\theta_{d}$, namely regular near polygons. We conclude our paper with the following open question.

Question When is a pseudo 1-homogeneous property (corresponding to one or more edges) implying the 1-homogeneous property?

We will most probably not get away with an algebraic condition, but we will need a combinatorial one.

\section{References}

1. Bannai, E., Ito, T.: Algebraic Combinatorics I: Association Schemes. Benjamin-Cummings, California (1984)

2. Brouwer, A.E., Cohen, A.M., Neumaier, A.: Distance-Regular Graphs. Springer, Berlin, Heidelberg (1989)

3. Curtin, B., Nomura, K.: 1-homogeneous, pseudo-1-homogeneous, and 1-thin distance-regular graphs. J. Comb. Theory Ser. B 93, 279-302 (2005)

4. Go, J.T., Terwilliger, P.M.: Tight distance-regular graphs and the subconstituent algebra. Eur. J. Comb. 23, 793-816 (2002)

5. Godsil, C.D.: Algebraic Combinatorics. Chapman and Hall, New York (1993)

6. Jurišić, A., Koolen, J.: A local approach to 1-homogeneous graphs. Des. Codes Cryptogr. 21, 127-147 (2000)

7. Jurišić, A., Koolen, J., Terwilliger, P.: Tight Distance-Regular Graphs. J. Algebr. Comb. 12, 163-197 (2000)

8. Pascasio, A.A.: Tight graphs and their primitive idempotents. J. Algebr. Comb. 10, 47-59 (1999)

9. Terwilliger, P.M.: The subconstituent algebra of a distance-regular graph; thin modules with endpoint one. Linear Algebra Appl. 356, 157-187 (2002)

10. Terwilliger, P.M.: An inequality involving the local eigenvalues of a distance-regular graph. J. Algebr. Comb. 19, 143-172 (2004)

11. Terwilliger, P.M., Weng, C.: Distance-regular graphs, pseudo primitive idempotents, and the Terwilliger algebra. Eur. J. Comb. 25(2), 287-298 (2004) 